\title{
Strongly Possible Functional Dependencies for SQL*
}

\author{
Munqath Alattar ${ }^{a b}$ and Attila Saliac
}

\begin{abstract}
Missing data is a large-scale challenge to research and investigate. It reduces the statistical power and produces negative consequences that may introduce selection bias on the data. Many approaches to handle this problem have been introduced. The main approaches suggested are either missing values to be ignored (removed) or imputed (filled in) with new values [14]. This paper uses the second method. Possible worlds and possible and certain keys were introduced in $[22,25]$, while certain functional dependencies (c-FD) were introduced in [23] as a natural complement to Lien's class of possible functional dependencies (p-FD) by [26], and Weak and strong functional dependencies were studied in [25]. The intermediate concept of strongly possible worlds introduced in a preceding paper [3] and results in strongly possible keys (spKey's) and strongly possible functional dependencies (spFD's) were studied. Also, a polynomial algorithm to verify a single spKey was given and it was shown that it is NP-complete in general to verify an arbitrary collection of spKeys. Furthermore, a graph-theoretical characterization was given for validating a given spFD $X \rightarrow_{s p} Y$.

We show, that the complexity to verify a single strongly possible functional dependency is NP-complete in general, then we introduce some cases when verifying a single spFD can be done in polynomial time. As a step toward axiomatization of spFD's, the rules given for weak/strong and certain functional dependencies are checked. Appropriate weakenings of those that are not sound for spFD's are listed. The interaction between spFD's and spKey's and certain keys is studied. Furthermore, a graph theoretical characterization of implication between singular attribute spFD's is given.
\end{abstract}

${ }^{*}$ Research of the second author was partially supported by the National Research, Development and Innovation Office (NKFIH) grants K-116769, K-132696 and SNN-135643. This work is also supported by the National Research, Development and Innovation Fund (TUDFO/51757/2019ITM, Thematic Excellence Program), the BME NC TKP2020 grant of NKFIH Hungary and it is also connected to the scientific program of the "Development of quality-oriented and harmonized $\mathrm{R}+\mathrm{D}+\mathrm{I}$ strategy and functional model at BME" project, supported by the New Hungary Development Plan (Project ID: TÁMOP-4.2.1/B-09/1/KMR-2010-0002).

${ }^{a}$ Department of Computer Science and Information Theory, Budapest University of Technology and Economics, Hungary

${ }^{b}$ Information Technology Research and Development Center, University of Kufa, Iraq, E-mail: m.attar@cs.bme.hu, ORCID: 0000-0002-4673-4902

${ }^{c}$ Alfréd Rényi Institute of Mathematics, Budapest, Hungary, E-mail: sali.attila@renyi.hu, ORCID: 0000-0002-4837-6360 
Keywords: strongly possible functional dependencies, strongly possible keys NULL values, data imputation, matchings in bipartite graphs, list coloring

\section{Introduction}

Incomplete data in databases are allowed in many of the modern systems. For example, when raw data is collected from different sources in data warehousing, some of the attributes may be available in some of the sources and may not be in some others. For this reason, it is important to treat constraints over incomplete tables. Aliriza et al. [12] showed that encountering up to half of the data values missing is common. So using analysis methods that work only with complete data tables makes mining the data more complicated.

Incompleteness occurs in database tables for different reasons. Date [10] identified seven types of NULL's as follows: value not applicable, value unknown, value does not exist, value undefined, value not valid, value not supplied, and value is the empty set. We consider the second, third, and seventh types as this paper deals with data consumption in an incomplete database table. We consider the symbol N/A for the other types of missing data and we assume it belongs to each domain and it is considered as a regular domain value for comparisons and analysis.

Missing values problem complicates data analysis. It also causes a loss of data efficiency and effectiveness [14]. Although some data analysis approaches get over the problem of incomplete databases, still most approaches require complete databases. To overcome the missing data problem, there are mainly two main methods, either ignoring the incomplete tuples or filling them with domain values chosen by some heuristics. [14].

Possible worlds, introduced by Köhler et al. [22], are obtained by replacing each missing data value with a value from its attribute's domain, which can be infinite. For an incomplete table, every possible world is a complete table that may contain some duplicated tuples. They defined a possible (certain) keys as a key that are satisfied by some (every) possible world of a (non-total) database table. For example, the table in Table 2a satisfies the possible key $\{$ Course Name $\}$ as there is a possible world that satisfies it, and every possible world of the table satisfies the certain key $\{$ Course Name, Year, Semester $\}$. Furthermore, no possible world of the table satisfies the key $\{$ Lecturer $\}$. Weak (strong) functional dependencies were defined by [25] as FD's that are satisfied by some (every) possible world. The table in Table 1a satisfies the strong FD $\square($ Name mrg_status $\rightarrow$ gender $)$ because every possible world satisfies it, and the weak FD $\diamond($ mrg_status $\rightarrow$ gender $)$, as there exist some possible worlds, but not all, satisfying the implication.

Most often, especially when the attribute's domain is not known, there is no reason to consider any other attribute value than the already existing values of the table. Types of cars, diagnoses of patients, applied medications, dates of exams, course descriptions, etc, are some examples. For that, the strongly possible world is 
Table 1: Possible and Strongly Possible Worlds

(a) Incomplete Table

\begin{tabular}{cccc}
\hline Name & gender & mrg_status & age \\
\hline$\perp$ & female & married & 32 \\
Sarah & female & $\perp$ & $\perp$ \\
David & $\perp$ & divorced & 38 \\
James & male & single & $\perp$ \\
James & male & widower & 47 \\
\hline
\end{tabular}

(b) Possible World

\begin{tabular}{cccc}
\hline Name & gender & mrg_status & age \\
\hline 30 & female & married & 32 \\
Sarah & female & lawyer & high \\
David & Apple & divorced & 38 \\
James & male & single & -12 \\
James & male & widower & 47 \\
\hline
\end{tabular}

(c) Strongly Possible World

\begin{tabular}{cccc}
\hline Name & gender & mrg_status & age \\
\hline David & female & married & 32 \\
Sarah & female & single & 32 \\
David & male & divorced & 38 \\
James & male & single & 38 \\
James & male & widower & 47 \\
\hline
\end{tabular}

defined as a possible world achieved by substituting each occurrence of NULL with a value from the corresponding attribute's existing values [3]. Strongly possible $\operatorname{key}(\mathrm{FD})$ is a $\operatorname{key}(\mathrm{FD})$ that satisfied by a strongly possible world. Values that are shown in each attribute of a table represent a part of that attribute's domain. When the attribute domain is unknown, choosing values out of its real domain to fill in a NULL may distort the data. For example, it would be unsuitable using value other than the ones appearing in the marriage status attribute to fill the $\perp$ in the second row in Table 1a. Other values like numbers, symbols, or any other strings with distant meanings may cause distortion. Therefore, a more meaningful and 
semantically acceptable possible (strongly possible) world would be provided using one of the already shown values in the attribute. As the possible world in Table 1c is preferred to the one in Table 1b. Strongly possible worlds were introduced by [3] where strongly possible keys (spKey's) were studied. The natural extension to strongly possible functional dependencies was investigated in [4].

\subsection{Contributions}

In the present paper, we study spFD's that were earlier introduced in $[4,5]$ as a generalized version of functional dependency constraint using the visible domain concept and we continue the work started in [3]. We provided a graph-theoretical condition for the spFD satisfaction in [4]. We give a list of axioms of weak/strong and certain FD's that are sound for spFD's, as well and describe several possible sound weakenings for those that are not sound. Interactions between spKeys and strongly possible FDs/certain FDs are investigated, and also the spFD's between singular attributes are studied. The main contributions of this paper are as follows:

- We analysed the properties of strongly possible keys and introduced a (worstcase exponential time) algorithm to verify a single spKey in [3]. A polynomialtime solution for the same problem is given and we show that verifying an arbitrary system of strongly possible keys is NP-complete, thus resolving an open problem from [3].

- A graph-theoretical characterization was given in [4] to verify when spFD $X \rightarrow_{s p} Y$ holds in an SQL table $T$ containing NULL's. We use that here to show that the satisfaction problem for a single spFD $X \rightarrow_{s p} Y$ is NPcomplete.

- We give a list of the axioms of Weak/Strong and Certain FD's that are sound for spFD's, with several possible weakenings or restrictions that keep soundness for those that are not sound.

- An interaction between the spKeys and sp/c-FDs is studied and the weakening and transitivity rules are introduced.

- We analyzed the case when the spFD's are restricted to singular attributes. A complete characterisation of the implication problem of this special case is given based on the fact that all the possible extensions of any NULL occurrences are shown in the table.

\subsection{Paper structure}

The organization of this paper is as follows. Section 2 contains the necessary definitions. Section 3 discusses related work. Algorithmic and complexity questions of strongly possible keys and strongly possible functional dependencies are introduced in Section 4. In section 5, some spFD's implication properties are studied. First the case if one side of an spFD is fixed is treated. Then sound implication rules for 
spFD's are listed. The proof of their correctness are given in [5]. Then the special case of spFD's between single attributes is treated using graph theoretical methods. Finally, Section 6 contains concluding remarks and future research directions.

\section{Definitions}

In this section, we recall some basic definitions introduced in [4]. Let $R=$ $\left\{A_{1}, A_{2}, \ldots A_{n}\right\}$ be a relation schema. The set of all the possible values for each attribute $A_{i} \in R$ is called the domain of $A_{i}$ and denoted by $D_{i}=\operatorname{dom}\left(A_{i}\right)$ for $i=$ $1,2, \ldots n$.

An instance $T=\left(t_{1}, t_{2}, \ldots t_{s}\right)$ over $R$ is a list of tuples that each tuple is a function $t: R \rightarrow \bigcup_{A_{i} \in R} \operatorname{dom}\left(A_{i}\right)$ and $t\left[A_{i}\right] \in \operatorname{dom}\left(A_{i}\right)$ for all $A_{i}$ in $R$. Note that this definition of tuples emphasizes that the order of the attributes in schema $R$ is irrelevant and by taking a list of tuples we use the bag semantics that allows several occurrences of the same tuple. For a tuple $t_{r} \in T$ and $X \subset R$, let $t_{r}[X]$ be the restriction of $t_{r}$ to $X$.

It is assumed that $\perp$ is an element of each attribute's domain that denotes missing information. $t_{r}$ is called $V$-total for a set $V$ of attributes if $t_{r}[A] \neq \perp$, $\forall A \in V$. Also a tuple $t_{r}$ is a total tuple if it is a $R$-total. $t_{1}$ and $t_{2}$ are weakly similar on $X \subseteq R$ denoted as $t_{1}[X] \sim_{w} t_{2}[X]$ defined by Köhler et al. [22] if:

$$
\forall A \in X \quad\left(t_{1}[A]=t_{2}[A] \text { or } t_{1}[A]=\perp \text { or } t_{2}[A]=\perp\right) .
$$
if:

Furthermore, $t_{1}$ and $t_{2}$ are strongly similar on $X \subseteq R$ denoted by $t_{1}[X] \sim_{s} t_{2}[X]$

$$
\forall A \in X \quad\left(t_{1}[A]=t_{2}[A] \neq \perp\right) .
$$

For the sake of convenience, we write $t_{1} \sim_{w} t_{2}$ if $t_{1}$ and $t_{2}$ are weakly similar on $R$ and use the same convenience for strong similarity. Thus, if $t_{1}$ and $t_{2}$ are both weakly similar to a NULL-free tuple $t$, then $t_{1} \sim_{w} t_{2}$. This is true because both of the non-total tuples $t_{1}$ and $t_{2}$ could be extended to be equal to the total tuple $t$.

Let $T=\left(t_{1}, t_{2}, \ldots t_{s}\right)$ be a table instance over $R . T^{\prime}=\left(t_{1}^{\prime}, t_{2}^{\prime}, \ldots t_{s}^{\prime}\right)$ is a possible world of $T$, if $t_{i} \sim_{w} t_{i}^{\prime}$ for all $i=1,2, \ldots s$ and $T^{\prime}$ is completely NULL-free. That is, we replace the occurrences of $\perp=t\left[A_{i}\right]$ with a value from the domain $D_{i}$ different from $\perp$ for all tuples and all attributes.

Weak functional dependency $\diamond X \rightarrow Y$ holds in $T$ if there exists a possible world $T^{\prime}$ such that $T^{\prime} \models X \rightarrow Y$ in the classical sense, that is functional dependency $X \rightarrow Y$ holds in $T^{\prime}$ meaning that if $t_{i}^{\prime}[X]=t_{j}^{\prime}[X]$ then $t_{i}^{\prime}[Y]=t_{j}^{\prime}[Y]$ is satisfied, for all pairs of tuples $t_{i}^{\prime}, t_{j}^{\prime} \in T^{\prime}$. Strong functional dependency $\square X \rightarrow Y$ holds in $T$ if functional dependency $X \rightarrow Y$ holds in all possible worlds $T^{\prime}$ of $T$. $X$ is a possible key if there exists a possible world $T^{\prime}$ such that $X$ is a key in $T^{\prime}$, and $X$ is a certain key if it is a key in every possible world of $T$. The following was proven in [22].

Theorem 1. $X \subseteq R$ is a certain (possible) key iff

$$
\forall t_{1}, t_{2} \in T: t_{1}[X] \nsim_{w} t_{2}[X]\left(t_{1}[X] \varkappa_{s} t_{2}[X]\right) .
$$


Table 2: Complete and Incomplete Datasets

(a) Incomplete Dataset

\begin{tabular}{lcccc}
\hline Course Name & Year & Lecturer & Credits & Semester \\
\hline Mathematics & 2019 & $\perp$ & 5 & 1 \\
Datamining & 2018 & Sarah & 7 & $\perp$ \\
$\perp$ & 2019 & Sarah & $\perp$ & 2 \\
\hline
\end{tabular}

(b) Complete Dataset

\begin{tabular}{lcccc}
\hline Course Name & Year & Lecturer & Credits & Semester \\
\hline Mathematics & 2019 & Sarah & 5 & 1 \\
Datamining & 2018 & Sarah & 7 & 2 \\
Datamining & 2019 & Sarah & 7 & 2 \\
\hline
\end{tabular}

\subsection{Strongly possible worlds}

The concepts of visible domain and strongly possible world were introduced in [3].

Definition 1. The visible domain of an attribute $A_{i}\left(V D_{i}\right)$ is the set of all distinct values except $\perp$ that are already used by tuples in $T$ :

$$
V D_{i}=\left\{t\left[A_{i}\right]: t \in T\right\} \backslash\{\perp\} \text { for } A_{i} \in R
$$

For example, the visible domain of the Credits attribute in Table 2a is $\{5,7\}$. So, for any dataset with no information about the attributes' domains, we define their structure and domains by the data itself. It is more reliable and realistic when considering only what information we have in a given dataset and depending on extracting the relationship between data to overcome the missing data problem. Strongly possible worlds are obtained by using the visible domain values in place of the occurrence of NULL's.

Definition 2. A possible world $T^{\prime}$ of $T$ is called strongly possible world (spWorld) if $t^{\prime}\left[A_{i}\right] \in V D_{i}$ for all $t^{\prime} \in T^{\prime}$ and $A_{i} \in R$.

We defined strongly possible keys (spKey's) and strongly possible functional dependencies (spFD's) in $[3,4]$ respectively by strongly possible worlds as follows.

Definition 3. Strongly possible functional dependency $X \rightarrow_{s p} Y$ holds in table $T$ over schema $R$ if there exists an spWorld $T^{\prime}$ of $T$ such that $T^{\prime}=X \rightarrow Y$. $X$ is a strongly possible key if there exists an spWorld $T^{\prime}$ of $T$ such that $X$ is a key in $T^{\prime}$, in notation $s p\langle X\rangle$. Note that this is not equivalent to $s p F D X \rightarrow_{s p} R$ since we use the bag semantics. 
In Table 2a, the spKey of the two attributes \{Course Name, Year is satisfied, and so is the spFD CourseName $\rightarrow_{s p}$ Semester, as the strongly possible world in Table $2 \mathrm{~b}$ shows it.

For a schema $R$, the NULL-free subschema (NFS) is a subset $R_{S} \subseteq R$ that corresponds to SQL's NOT NULL constraint. A table $T$ over $R$ satisfies NFS $R_{S}$ if it is $R_{S}$-total, that means every tuple $t \in T$ is $R_{S}$-total $\left(\forall A \in R_{S}: t[A] \neq \perp\right)$. If $T$ satisfies NFS $R_{S}$, then we say $T$ is over $\left(R, R_{s}\right)$. Also, if $\Sigma$ is a set of integrity constraints (for example spFD's), then a table $T$ over $\left(R, R_{s}\right)$ is an Armstrong instance of $\Sigma$ if

(1) $T \models \sigma \Longleftrightarrow \Sigma \models \sigma$ for any constraint $\sigma$, and

(2) $\forall A \in R \backslash R_{S}, \exists t \in T$ such that $t[A]=\perp$.

This is the classical Armstrong instance extended by requiring that if an attribute is not in the NULL-free subschema, then it certainly contains NULL's.

\section{Related work}

Keys and functional dependencies are two main constraints that enforce the semantics of relational databases. Database tables of real database systems usually contain occurrences of null values and for some cases, this happens in candidate key columns. Many studies have been done on handling the missing values problem.

Aliriza et.al. introduced a framework of imputation methods in [14] and evaluated how the selection of different imputation methods affected the performance in [12]. Experimental analyses of several algorithms for imputation of missing values were provided by $[16,13,2,7]$. An approach introduced by Zhang et.al. discussed and compared different approaches that use only known values [29].

Sree [11] suggests that it is necessary to substitute the missing values with values based on other information in the dataset to overcome the biased effects that affect the accuracy of classification. Likewise, for each NULL in an attribute, we use the attribute's existing values. Cheng et.al.[8] used a clustering algorithm to cluster data and calculate coefficient values between different attributes producing the minimum average error.

Interactions of functional dependencies and other integrity constraints with null values have long been studied. Early investigations focused on "fixing" the database using the Chase procedure, such as Grahne did in [15]. Imlienski and Lipski [19] also investigated the properties of Chase concerning NULL's. Kiss and Márkus [21], provided a chase procedure for functional and inclusion dependencies to provide graph manipulation rules for dependency inference.

The two most used interpretations of NULL's are "value unknown at present" and "no information". The first one leads to possible world semantics that is NULL's are substituted by domain values to obtain total tables. A three-valued model of FD satisfaction is given by Vassilou [27]. It takes into account that all possible words of a table $T$ are considered and a functional dependency either holds, does 
not hold or may hold on $T$. This latter means that in some possible worlds it holds, and in some other ones it does not hold. Fuzzyness is naturally associated with imperfect information. For fuzzy relational databases, the truth value of FD's was studied by Achs and Kiss in [1] Levene and Loizou defined weak and strong functional dependencies based on possible world concept. A weak FD holds in some of the possible worlds and a strong FD holds in all possible worlds. A sound and complete axiom system is given for them in [25].

Both of unknown, as well as non-existing data can be treated by the "no information" approach. Lien [26] defined functional dependencies that hold if strong similarity on the LHS implies equality on RHS. The equality here means that when two tuples are equal on an attribute, if the attribute value is NULL in one tuple, then the other tuple must also be NULL in the same attribute. This dependency is same as the possible FD (p-FD) of Köhler and Link [23]. The novelty of the latter paper is the concept of certain functional dependencies. A c-FD holds if the weak similarity on LHS implies equality on the RHS, and the equality here is defined in the same way as the equality of attribute values on RHS of p-FD's. An axiom system for functional dependencies with NULL's was given by Atzeni and Morfuni [6], but the drawback was that they allowed no NULL's on the LHS.

Levene and Loizou introduced weak and strong FD's, where weak (strong) functional dependency is satisfied if it holds in some (every) possible world [25]. We also used the possible world semantics to introduce our strongly possible functional dependency and it fits between weak and strong FD's. Let suppose we have a table instance with at least one non-NULL value is there in each attribute, then a c-FD $X_{w} \rightarrow Y$ satisfaction implies the satisfaction of the spFD $X \rightarrow_{s p} Y$. But the satisfaction of $X \rightarrow_{s p} Y$ does not imply the satisfaction of the p-FD $X_{s} \rightarrow Y$. For example, Year $\rightarrow_{s p}$ Credits is satisfied in Table2a, while Year ${ }_{s} \rightarrow$ Credits is violated. Example 1 shows a brief comparison of the different functional dependencies. This example is shown in [4], we repeat it here after fixing a typo appearing in the conference version.

Example 1. Let $T$ be the following SQL table.

\begin{tabular}{llll}
\hline employee & dept & manager & salary \\
\hline Knuth & NULL & Chomsky & 100,000 \\
Turing & CS & von Neumann & NULL \\
Turing & NULL & Gödel & NULL \\
\hline
\end{tabular}

In the following table, we compare the six types of FD's shown in the SQL table: 3 -valued [27], weak and strong [25], possible [26], certain [23] and strongly possible functional dependencies (spFD's). 


\begin{tabular}{lllllll}
\hline & 3-valued & weak & strong & possible & certain & spFD \\
\hline$e \rightarrow d$ & unknown & $\mathrm{T}$ & $\mathrm{F}$ & $\mathrm{F}$ & $\mathrm{F}$ & $\mathrm{T}$ \\
$e \rightarrow m$ & $\mathrm{~F}$ & $\mathrm{~F}$ & $\mathrm{~F}$ & $\mathrm{~F}$ & $\mathrm{~F}$ & $\mathrm{~F}$ \\
$e \rightarrow s$ & unknown & $\mathrm{T}$ & $\mathrm{F}$ & $\mathrm{T}$ & $\mathrm{T}$ & $\mathrm{T}$ \\
$d \rightarrow d$ & $\mathrm{~T}$ & $\mathrm{~T}$ & $\mathrm{~T}$ & $\mathrm{~T}$ & $\mathrm{~F}$ & $\mathrm{~T}$ \\
$d \rightarrow m$ & unknown & $\mathrm{T}$ & $\mathrm{F}$ & $\mathrm{T}$ & $\mathrm{F}$ & $\mathrm{F}$ \\
$m \rightarrow e$ & $\mathrm{~T}$ & $\mathrm{~T}$ & $\mathrm{~T}$ & $\mathrm{~T}$ & $\mathrm{~T}$ & $\mathrm{~T}$ \\
$m \rightarrow d$ & $\mathrm{~T}$ & $\mathrm{~T}$ & $\mathrm{~T}$ & $\mathrm{~T}$ & $\mathrm{~T}$ & $\mathrm{~T}$ \\
\hline
\end{tabular}

Possible and certain keys were introduced by Köhler et. al. [22], such that a set $K$ of attributes is a possible (certain) key if it is a key in some (every) possible world. The "strongly possible" concept of the present paper is in between of these two, where a strongly possible world is a possible world also. As possible worlds may use any value from an attribute domain to substitute a NULL it may allow an infinite set of values. On the other hand, strongly possible worlds allow only a finite set of values and created from finite attribute domains. Some of the results in [22] assume that some attribute domains are infinite. In this paper, we study the dependencies without that assumption.

Imielinski [18] introduced the construction of OR-relations which is another closely related concept to our spFD's. An OR-tuple over schema $R\left(A_{1}, A_{2}, \ldots, A_{n}\right)$ is a mapping $t: R \rightarrow \cup_{i=1}^{n} \mathcal{P}_{0}\left(\operatorname{dom}\left(A_{i}\right)\right)$ such that $t\left[A_{i}\right] \in \mathcal{P}_{0}\left(\operatorname{dom}\left(A_{i}\right)\right)$, where $\mathcal{P}_{0}(X)$ is the collection of finite subsets of set $X$. An OR-relation $r$ is a bag of ORtuples and a possible world $r^{\prime}$ for $r$ is a collection of tuples $t^{\prime}$ such that $t^{\prime}\left[A_{i}\right] \in t\left[A_{i}\right]$ for all $t \in r$. A wFD $\diamond X \rightarrow Y$ is satisfied by an OR-relation $r$ iff there is a possible world $r^{\prime}$ of $r$ such that $r^{\prime} \models X \rightarrow Y$. Hartmann and Link already noted in [17] that Union rule does not hold for wFD's in OR-relations. Our strongly possible world concept can be modelled by OR-relations in such a way that if $t\left[A_{i}\right]=\perp$ for some tuple in an SQL table $T$, then $t\left[A_{i}\right]$ is replaced by the OR-set $V D\left(A_{i}\right)$, the visible domain of $A_{i}$. So strongly possible worlds and spFD's can be considered as special cases of possible worlds of OR-relations and wFD's in them. Our analysis shows, that most of the classical implication rules, axioms do not hold even in this special case.

Finally, Wei and Link [28] aim to define a solid generalization of functional dependencies that do not rely on the interpretation of NULL's. Also, the two papers $[23,28]$ studied the databases normalization based on the appropriate functional dependencies. It is a future research topic on how our spFD's could be applied for normalization, as normalization is important to eliminate redundancy that may cause inconsistency at updates. 


\section{Algorithmic and complexity questions}

In this section the complexity questions connected to strongly possible keys and strongly possible functional dependencies are studied. The main problems are NPcomplete in general, but several important special cases can be solved in polynomial time.

\subsection{Strongly possible keys}

Following is the algorithmic question we study here. Let $T$ be a SQL table and $\Sigma$ be a collection of strongly possible key constraints, does $T \models \Sigma$ hold? We may assume, without loss of generality, that there exists at least one spWorld for every treated table. This is a reasonable assumption, as the non-existence of an spWorld happens only if a table contains only NULL's in an attribute.

In [3], an algorithm is given using bipartite matchings for the case when a single spKey needs to be checked, i.e, $\Sigma=\{s p\langle K\rangle\}$. If $K=\left\{A_{1}, A_{2} \ldots A_{b}\right\}$, then the running time of that algorithm is $O\left(|R|\left(|T|+\left|T^{\star}\right|\right)\right)+O\left(\left(|T|+\left|T^{\star}\right|\right)|E|\right)$, where $T^{\star}$ is the set of total tuples $T^{\star}=\left\{t^{\star} \in \Pi_{i=1}^{b} V D_{i}: \exists t \in T\right.$ such that $\left.t[K] \sim_{w} t^{\star}[K]\right\}$. However, the size of $T^{\star}$ can be an exponential function of size of $T$. In [4], we gave a polynomial-time refinement of that algorithm. The refined algorithm states that if a single spKey $s p\langle K\rangle$ is to be checked, then considering $\left.T\right|_{K}$ is enough, because of $K$ is a key if and only if the tuples are pairwise distinct on $K$. Next proposition was introduced in [3], it gave a sufficient condition to determine the satisfaction of a given spKey. Let us assume that $T^{\star} \subseteq V D_{1} \times V D_{2} \times \ldots \times V D_{b}$ is defined by $T^{\star}=\left\{t^{\star} \in V D_{1} \times V D_{2} \times \ldots \times V D_{b}: \exists t \in T: t[K] \sim_{w} t^{\star}\right\}$, and define a bipartite graph $G=\left(T, T^{\star} ; E\right)$ with $\left\{t, t^{\star}\right\} \in E \Longleftrightarrow t[K] \sim_{w} t^{\star}[K]$. Note that we use here the standard bipartite graph notation, $T$ and $T^{\star}$ are the partite classes. Propositions 1, 2 and 3, Theorem 2, and Algorithm 1 were proven in [4]. We repeat them here to be self-contained.

Proposition 1. $T \models s p\langle K\rangle$ holds if and only if there exists a matching in $G=$ $\left(T, T^{\star} ; E\right)$ covering $T$.

We may resort to generating only part of $T^{\star}$ to ensure that the algorithm will run in polynomial time. Let $T=\left\{t_{1}, t_{2} \ldots t_{m}\right\}$ and $\ell\left(t_{i}\right)=\mid\left\{t^{\star} \in V D_{1} \times V D_{2} \times \ldots \times\right.$ $\left.V D_{b}: t^{\star} \sim_{w} t_{i}[K]\right\} \mid$. Note that $\ell\left(t_{i}\right)=\prod_{j: t_{i}\left[A_{j}\right]=\perp}\left|V D_{j}\right|$, then these values can be calculated by scanning $T$ once and using appropriate search tree data structures to hold values of visible domains of each attribute. Sort tuples of $T$ in non-decreasing $\ell\left(t_{i}\right)$ order, i.e. assume that $\ell\left(t_{1}\right) \leq \ell\left(t_{2}\right) \leq \ldots \leq \ell\left(t_{p}\right)$. Let $j=\max \left\{i: \ell\left(t_{i}\right)<i\right\}$ and $T_{j}=\left\{t_{1}, t_{2}, \ldots t_{j}\right\}$, and furthermore, $T_{j}^{\star}=\left\{t^{\star}: \exists t \in T_{j}: t^{\star} \sim_{w} t[K]\right\} \subseteq$ $V D_{1} \times V D_{2} \times \ldots \times V D_{b}$. Note that $\left|T_{j}^{\star}\right| \leq \frac{1}{2} j(j-1)$. If $\forall i=1,2, \ldots, m: \ell\left(t_{i}\right) \geq i$, then define $j=0$ and $T_{j}^{\star}=\emptyset$.

Proposition 2. $T \models s p\langle K\rangle$ holds if and only if $j=0$ or there exists a matching in $G^{\prime}=\left(T_{j}, T_{j}^{\star} ;\left.E\right|_{T_{j} \times T_{j}^{\star}}\right)$ covering $T_{j}$. 
Proposition 2 was proven in [4] and it gives the basis of a polynomial-time algorithm to determine whether $T \models s p\langle K\rangle$ holds or not.

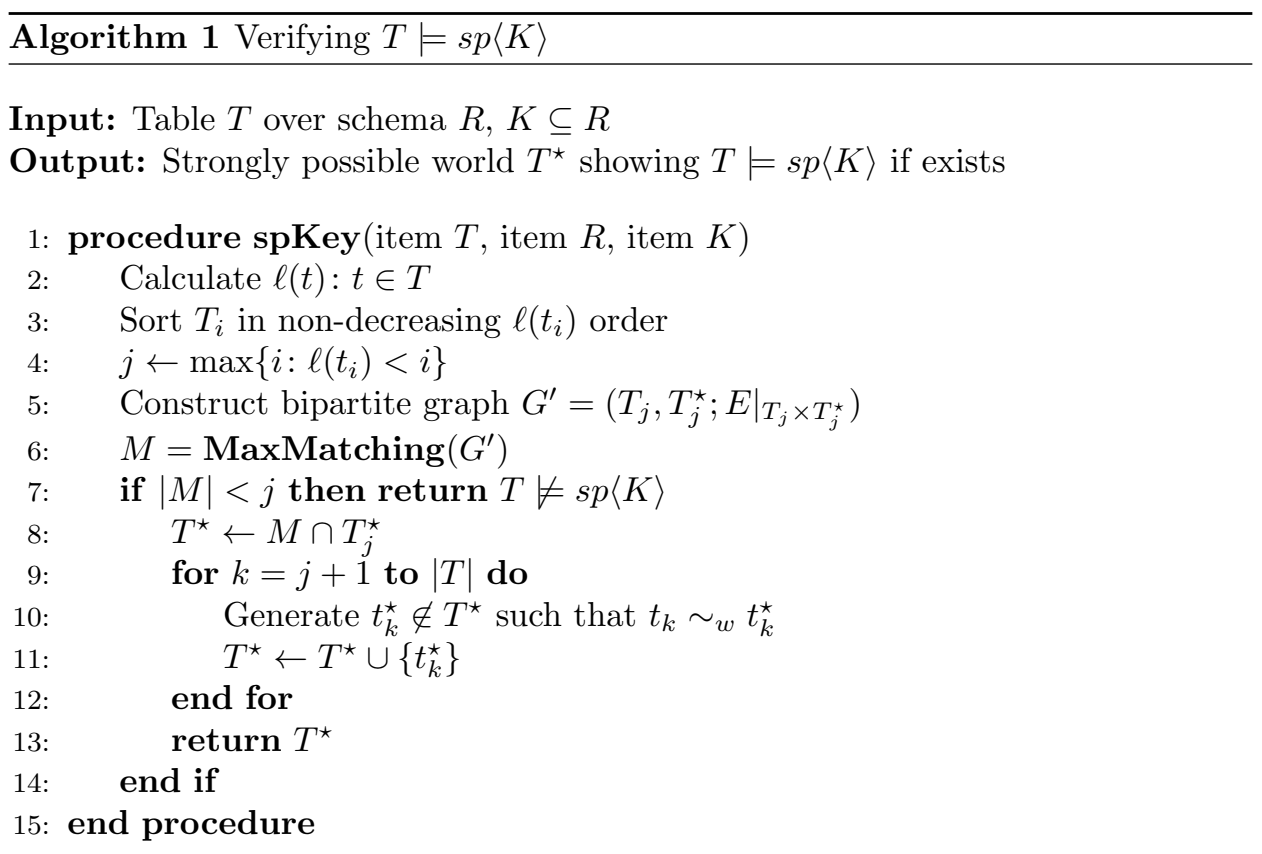

Algorithm 1 was introduced in [4] and its running time is $O(|K| \cdot|T| \log |T|+$ $\left.|T|^{5}\right)$. Theorem 2 shows that the spKey problem is NP-complete in general, and its proof is in [4]. We reduced the problem of deciding whether $T=\Sigma$ for a collection $\Sigma$ of spKey constraints to the problem of finding the maximal common independent set of three or more matroids in [3]. This matroid intersection problem is NPcomplete, however the reduction given was not one-to-one, so it did not prove, just hinted the NP-completeness of spKey problem. Finally, by modifying an argument of [22], we could prove the following theorem in [4] by a Karp-reduction of 3SAT to our problem.

Theorem 2. The strongly possible key satisfaction problem is NP-complete.

In some special cases, $T \models \Sigma$ can be verified in polynomial time, where $\Sigma$ is a collection of strongly possible key constraints, as the following Proposition shows.

Proposition 3. Let us assume that $T$ is a table over schema $R$, furthermore let $\Sigma=$ $\left\{s p\left\langle K_{1}\right\rangle, s p\left\langle K_{2}\right\rangle, \ldots, s p\left\langle K_{m}\right\rangle\right\}$ be a collection of spKey constraints. If $K_{i} \cap K_{j}=\emptyset$ for $i \neq j$, then $T \models \Sigma$ can be decided in polynomial time. 


\subsection{Graph theoretical characterization of strongly possible functional dependencies}

In this section, we introduce a graph-theoretical characterization that determines when $T \models X \rightarrow_{s p} Y$. Recall that for a table $T$ over Schema $R T \models X \rightarrow_{s p}$ $Y$ if and only if there exists an spWorld $T^{\prime}$ of $T$ such that $T^{\prime} \models X \rightarrow Y$. If $T=\left\{t_{1}, t_{2}, \ldots, t_{p}\right\}$ and $T^{\prime}=\left\{t_{1}^{\prime}, t_{2}^{\prime}, \ldots, t_{p}^{\prime}\right\}$ with $t_{i} \sim_{w} t_{i}^{\prime}$, then $t_{i}^{\prime}$ is called an sp-extension or in short an extension of $t_{i}$. Let $X \subseteq R$ be a set of attributes and let $t_{i} \sim_{w} t_{i}^{\prime}$ such that for each $A \in R: t_{i}^{\prime}[A] \in V D(A)$, then $t_{i}^{\prime}[X]$ is a strongly possible extension of $t_{i}$ on $X$. We exclude any attribute with all NULL values, because the visible domain for such attribute is the empty set. We recall the weak similarity graph [4], as a useful tool in investigations of strongly possible functional dependencies (spFD's in short).

Definition 4. Let $T=\left\{t_{1}, t_{2}, \ldots t_{p}\right\}$ be a table (instance) over schema $R$. The weak similarity graph $G_{Y}$ with respect to $Y \subseteq R$ is defined as $G_{Y}=(T, E)$, where $\left\{t_{i}, t_{j}\right\} \in E \Longleftrightarrow t_{i}[Y] \sim_{w} t_{j}[Y]$.

Theorem 3 characterizes using weak similarity graphs when $T \models X \rightarrow_{s p} Y$ holds. The theorem was proved in [4].

If $T \models X \rightarrow_{s p} Y$, then there exists an spWorld $T^{\prime}$ of $T$ such that $T^{\prime} \models X \rightarrow Y$. This latter one holds iff whenever $t_{1}^{\prime}[Y] \neq t_{2}^{\prime}[Y]$ then $t_{1}^{\prime}[X] \neq t_{2}^{\prime}[X]$ is also satisfied. Now, if $t_{1}, t_{2} \in T$ are such tuples that $t_{1}[Y] \chi_{w} t_{2}[Y]$, then certainly $t_{1}^{\prime}[Y] \neq t_{2}^{\prime}[Y]$ holds in any spWorld $T^{\prime}$. That is, in order to $T \models X \rightarrow_{s p} Y$ hold $t_{1}^{\prime}, t_{2}^{\prime}$ must also satisfy $t_{1}^{\prime}[X] \neq t_{2}^{\prime}[X]$. Recall that by Definition $4, t_{1}[Y] \neq_{w} t_{2}[Y]$ holds exactly when $\left\{t_{1}, t_{2}\right\}$ is an edge in the complement of the weak similarity graph $G_{Y}$. We may think about $t_{i}^{\prime}[X]$ 's as colors assigned to vertices of $\overline{G_{Y}}$ and then we obtain that this coloring must be proper. Now colors that can be assigned to vertices come from lists special to vertices, namely from the sets strongly possible extensions of $t$ on $X$. Let $T$ be a table over schema $R, t \in T$ and $X \subseteq R$. $t^{\prime}$ is a strongly possible extension of $t$ on $X$ if $t[X] \sim_{w} t^{\prime}[X], t^{\prime}$ is $X$-total and $\forall X_{i} \in X: t^{\prime}\left[X_{i}\right] \in V D_{X_{i}}$. The following theorem introduced in [4] characterizes when $T \models X \rightarrow_{s p} Y$ holds using weak similarity graphs. It tells us that the existence of proper coloring of $\overline{G_{Y}}$ using the lists determined by the strongly possible extensions on $X$ is a necessary and sufficient condition for $T \models X \rightarrow_{s p} Y$ to hold.

Let $G(V, E)$ be a graph and $L: V \rightarrow 2^{\mathbb{N}}$ be a mapping that assigns each vertex a list of colors $L(v)$. A list coloring of $G$ using lists $\{L(v): v \in V\}$ is a mapping $c: V \rightarrow \bigcup_{v \in V} L(v)$ such that $c(v) \in L(v)$ and $c(u) \neq c(v)$ if $\{u, v\} \in E$. We use Theorem 3 in proofs of sound interference rules.

Theorem 3. Let $T=\left\{t_{1}, t_{2} \ldots t_{m}\right\}$ be a table over schema $R$. For $X, Y \subseteq R$, $T \models X \rightarrow_{s p} Y$ holds iff $\overline{G_{Y}}$ can be list colored using lists $\left\{t_{i}^{1}, t_{i}^{2} \ldots t_{i}^{r_{i}}\right\}$ for $t_{i} \in T$, where $\overline{G_{Y}}$ is the complement of the weak similarity graph on $Y$ and $t_{i}^{j}$ 's are the strongly possible extensions of $t_{i}$ on $X$.

Table 3 illustrates that sets of tuples that are pairwise weakly similar on $Y$ form a weak similarity clique. Now, tuples from a given clique have a unique non-NULL 
value in each attribute of $Y$, unless they all contain NULL in that attribute. In both cases, there is a way to extend each tuple that tuples in the same clique become identical on $Y$. So those tuples that are in one weak similarity clique on $Y$ can be list colored by the same color on $X$ so that $T \models X \rightarrow_{s p} Y$. Note that weak similarity cliques of $G_{Y}$ are independent vertex sets in $\overline{G_{Y}}$.

Table 3: Color classes and weak similarity cliques.

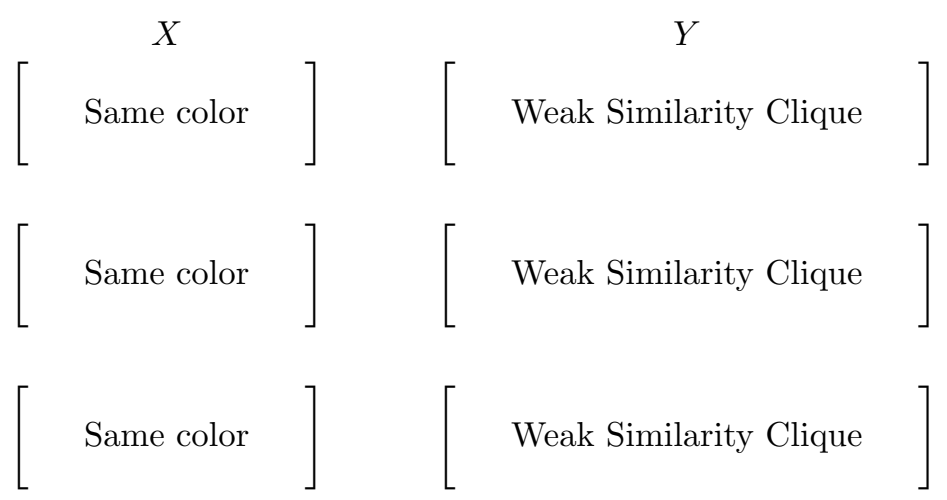

\subsection{Complexity of strongly possible functional dependencies}

List coloring problem is NP-complete even if all lists have length three [24]. This suggests that deciding whether a given spFD is satisfied in an SQL table is NPcomplete even if all the tuples have a maximum of three extensions. However, it is not obvious the "intractable cases" of list coloring problem really correspond to spFD-satisfaction, so we give a direct proof of NP-completeness.

Definition 5. The SPFD-SATISFACTION problem is defined as follows.

Input: An SQL-table $T$ over schema $R, X, Y \subseteq R$.

Question: Does $T \models X \rightarrow_{s p} Y$ hold?

Theorem 4. The SPFD-SATISFACTION problem is NP-complete.

Proof. SPFD-SAtisfaction is in NP, since an spWorld $T^{\prime}$ of $T$ such that $T^{\prime} \models$ $X \rightarrow Y$ is a good witness. It is clearly of polynomial size of the input and whether $T^{\prime} \models X \rightarrow Y$ holds can be checked in polynomial time by pairwise comparisons of tuples.

In order to prove that SPFD-SATISFACTION is NP-hard a Karp-reduction from 3-Color to SPFD-SAtisfaction is given. Let $G=(V, E)$ be an input of 3Color with $V=\left\{v_{1}, v_{2}, \ldots, v_{n}\right\}$. An SQL table $T$ is constructed over schema $R=\left\{A_{0}, A_{1}, \ldots, A_{n}\right\}$ of $n+1$ tuples and $X=\left\{A_{0}\right\}$ and $Y=\left\{A_{1}, A_{2}, \ldots, A_{n}\right\}$ is set so that the complement $\overline{G_{Y}}$ of the weak similarity graph on $Y$ is isomorphic 
to $G$ plus three isolated vertices. Let $t_{1}, t_{2}, \ldots, t_{n}$ be defined by induction on $i$ as follows. $t_{1}\left[A_{1}\right]=1$. If $t_{1}, t_{2}, \ldots, t_{i}$ are defined for $A_{1}, A_{2}, \ldots, A_{i}$, then let

$$
t_{i+1}\left[A_{j}\right]= \begin{cases}\perp & \text { if } 1 \leq j \leq i \\ 1 & \text { if } j=i+1\end{cases}
$$

furthermore, for $j=1,2, \ldots, i$

$$
t_{j}\left[A_{i+1}\right]= \begin{cases}2 & \text { if }\left\{v_{j}, v_{i+1}\right\} \in E(G) \\ \perp & \text { if }\left\{v_{j}, v_{i+1}\right\} \notin E(G)\end{cases}
$$

Finally, let $t_{n+k}$ be defined for $k=1,2,3$ as $t_{n+k}\left[A_{0}\right]=k, t_{n+k}\left[A_{j}\right]=\perp$ for $j=1,2, \ldots, n$. Obviously, $T$ can be constructed from $G$ in polynomial time. Our claim is that $G$ is 3 -colorable iff $T=X \rightarrow_{s p} Y$. Indeed, $t_{n+k}$ for $k=1,2,3$ are isolated vertices in $\overline{G_{Y}}$, so $\overline{G_{Y}}$ is list-colorable with extensions of $t_{j}$ over $X$ iff $\overline{G_{Y}}$ restricted to $W=\left\{t_{1}, t_{2}, \ldots, t_{n}\right\}$ is list-colorable. Observe that $\left.\overline{G_{Y}}\right|_{W}$ is isomorphic to $G=(V, E)$, Indeed, for $1 \leq i<j \leq n t_{i}[Y] \not_{w} t_{j}[Y]$ if $\left\{v_{i}, v_{j}\right\} \in E(G)$, because $t_{i}\left[A_{j}\right]=2$ and $t_{j}\left[A_{j}\right]=1$, on the other hand if $\left\{v_{i}, v_{j}\right\} \notin E(G)$, then $t_{i}\left[A_{j}\right]=\perp$ and $t_{j}\left[A_{j}\right]=1, t_{i}\left[A_{i}\right]=1$ and $t_{j}\left[A_{i}\right]=\perp$, furthermore $t_{i}\left[A_{\ell}\right], t_{j}\left[A_{\ell}\right] \in\{\perp, 2\}$ for $\ell \notin\{i, j\}$. Finally, $V D_{X}=\{1,2,3\}$, so the list of extensions of $t_{i}$ on $X$ is $\{1,2,3\}$ for all $1 \leq i \leq n+3$. Thus any proper list coloring of $\overline{G_{Y}}$ with extensions of $t_{i}$ on $X$ gives a 3 -coloring of $G$, and vice versa, any 3 -coloring of $G$ can easily be extended to a proper list coloring of $\overline{G_{Y}}$ with extensions of $t_{i}$.

However, this problem can be solved in polynomial time for some special cases. Complete graphs can be reduced to the verifying spKey problem, as the following proposition shows.

Proposition 4. $T \models X \rightarrow_{s p} Y$ can be decided in polynomial time if $\overline{G_{Y}}$ is a complete graph.

Proof. As $\overline{G_{Y}}$ is a complete graph, then the tuples are pairwise distinct by some non-NULL value on $Y$, then, all the tuples in $T$ should be pairwise distinctly colored on $X$ to satisfy the $\operatorname{spFD} X \rightarrow_{s p} Y$. That is, $T \models X \rightarrow_{s p} Y \Longleftrightarrow T \models s p\langle X\rangle$ that can be checked in polynomial time using Algorithm 1.

Greedy algorithm can be applied to find a proper list coloring of a graph $G=$ $(V, E)$ if for $\forall v \in V$ the list size of $v$ is at least $d_{G}(v)+1$. This can naturally be applied in our context, as well, that is $T \models X \rightarrow_{s p} Y$ can be decided in polynomial time if $\forall t \in T$, number of all extensions of $t$ on $X$ is larger than the number of weakly similar tuples to $t$ on $Y$.

In order to apply list coloring, first the lists should be generated. However, the number of $X$-extensions of $t$ of a tuple $t \in T$ may easily be exponential function of the size of the input as it was seen in Section4.1. This obstacle can be overcome by the following observation since it is enough to generate at most $\Delta\left(\overline{G_{Y}}\right)+1$ $X$-extensions for each tuple $t$. 
Proposition 5. There exists a list coloring with the lists generated for each tuple of size less than or equal $\Delta\left(\overline{G_{Y}}\right)+1$ if and only if there exists list coloring with full lists.

Proof. $\Rightarrow$ Indeed, same list coloring can be used.

$\Leftarrow$ Assume there exists list coloring with full lists. And let $\left\{t_{1}, t_{2}, \ldots, t_{r}\right\}$ be set of tuples with number of extensions on $X$ at most $\Delta\left(\overline{G_{Y}}\right)$ and let $\left\{t_{r+1}, t_{r+2}, \ldots, t_{s}\right\}$ be the set of other tuples. Take $\Delta\left(\overline{G_{Y}}\right)+1$ elements lists for $\left\{t_{r+1}, t_{r+2}, \ldots, t_{s}\right\}$. Then, keep the coloring of $\left\{t_{1}, t_{2}, \ldots, t_{r}\right\}$ and then color $\left\{t_{r+1}, t_{r+2}, \ldots, t_{s}\right\}$ using greedy algorithm.

If $\overline{G_{Y}}$ is a tree, the list coloring problem can be solved using dynamic programming techniques in polynomial time [20]. Generally, list coloring problem restricted to graphs of maximum degree two, such as cyclic graphs, is polynomially solvable [24].

Weak similarity graphs can be used on the LHS of an spFD in a special case. Namely, if components of weak similarity graph $G_{X}$ with respect to $X$ are cycles of length at least 4 , then $T \models s p\langle X\rangle$, in particular for any $Y \subseteq R, T \models X \rightarrow_{s p} Y$ holds.

Proposition 6. If each connected component $C$ of the weak similarity graph $G_{X}$ with respect to $X$ is a cycle of length $\geq 4$, then $s p\langle X\rangle$ holds.

Proof. Let $T=\left\{t_{1}, t_{2}, \ldots, t_{p}\right\}$ be a table over schema $R$ such that each component $C$ of the weak similarity graph $G_{X}$ with respect to $X$ is a cycle of length $\geq 4$. We need NULL-free tuples from $t_{i}^{\prime} \in V D_{1} \times V D_{2} \times \ldots \times V D_{n}$ such that $t_{i}[X] \sim_{w} t_{i}^{\prime}[X]$ and $t_{i}^{\prime}[X]$ 's are pairwise distinct in order to $s p\langle X\rangle$ hold. Since two tuples can only have identical extensions on $X$ if they are weakly similar on $X$, it is enough to construct $t_{i}^{\prime}[X]$ 's for each component of $G_{X}$ separately. If this single component is a circle $\left(t_{1}[X] \sim_{w} t_{2}[X] \sim_{w} t_{3}[X] \sim_{w} \ldots \sim_{w} t_{k}[X] \sim_{w} t_{1}[X]\right)$, then any extension of $t_{2}$ is distinct on $X$ from any extensions of $t_{4} \ldots t_{k}$. There exist $A \in X$ such that $t_{1}[A] \neq t_{3}[A]$ and both $t_{1}[A], t_{3}[A] \neq \perp$, because $t_{1}[X] \chi_{w} t_{3}[X]$. We need to make $t_{2}^{\prime}$ different from $t_{1}^{\prime}$ and $t_{3}^{\prime}$, so that we can set $t_{2}^{\prime}[A]=t_{3}[A]$ and this distinguishes it from $t_{1}^{\prime}$. Applying the same idea around the cycle, $t_{i}[X] \sim_{w} t_{i+1}[X] \sim_{w} t_{i+2}[X]$ will make $t_{i}^{\prime}$ and $t_{i+1}^{\prime}$ distinct.

\section{Implications among strongly possible functional dependencies}

This section treats the implication properties of spFD's. The cases when one side of the dependency is a fixed attribute set is characterized, then axioms and rules of weak, strong, possible and certain FD's are analysed with respect to spFD's. In addition to that, the interactions with different key concepts are treated. Finally, a complete characterization of the singular attribute case is given. 


\subsection{Strongly possible functional dependencies with one side fixed}

For $T=X \rightarrow_{s p} Y$, fixing the left-hand side of the spFD, makes the right-hand sides form a down-set, i.e, if $Y^{\prime} \subset Y$, then $T \models X \rightarrow_{s p} Y^{\prime}$ also holds. We introduced Proposition 7 in a proceedings paper [4], we repeat it here to fix an error in the proof of that version. It shows that for fixed left-hand side of spFD's, there is no other restriction for the right-hand sides than forming a down-set.

Proposition 7. Let $\left(R, R_{S}\right)$ be a schema and $X \nsubseteq R_{S}$. Let $\mathcal{Y}$ be a down-set of subsets of $R \backslash X$. Then there exists a table $T$ over $\left(R, R_{S}\right)$ such that $T \models X \rightarrow_{s p} Y$ holds iff $Y \cap(R \backslash X) \in \mathcal{Y}$.

Proof. Let the maximal elements of $\mathcal{Y}$ be $Y_{1}, Y_{2}, \ldots, Y_{s}$, that is $\mathcal{Y}=\left\{A: \exists i A \subseteq Y_{i}\right\}$ and $Y_{i} \nsubseteq \subseteq Y_{j}$ for $i \neq j$. Let $A_{0} \in X \backslash R_{S}$ be a fixed attribute, and $A_{1}, A_{2}, \ldots A_{n}$ be the other attributes of $R$. Table $T$ contains tuples $t_{0}, t_{1}, \ldots t_{s}$ such that

$$
t_{0}\left[A_{i}\right]=\left\{\begin{array}{l}
\perp \text { if } i=0 \\
0 \text { if } i>0
\end{array}\right.
$$

and

$$
t_{i}\left[A_{j}\right]=\left\{\begin{array}{c}
0 \text { if } A_{j} \in Y_{i} \cup X \\
i \text { if } A_{j} \notin Y_{i} \cup X
\end{array} \quad \text { for } i=1,2, \ldots, s \text { and } j=1,2, \ldots, n\right.
$$

\begin{tabular}{|c|c|c|c|c|}
\hline$A_{0}$ & $A_{1}$ & $\ldots$ & & $A_{n}$ \\
\hline$\perp$ & 0 & $\ldots$ & & 0 \\
\hline $\mathrm{i}$ & 0 & 0 & $\mathrm{i}$ & $\mathrm{i}$ \\
\hline
\end{tabular}

finally $t_{i}\left[A_{0}\right]=i$ for $i=1,2, \ldots, s$. So, the table is constructed as follows.

$$
Y_{i} \cup X
$$

$Y \in \mathcal{Y} \Longleftrightarrow \exists 1 \leq i_{Y} \leq s: Y \subseteq Y_{i_{Y}}$, so FD $X \rightarrow Y$ holds in the spWorld obtained by replacing $\perp$ in $t_{0}\left[A_{0}\right]$ by $i_{Y}$, because only tuples $t_{0}$ and $t_{i_{Y}}$ can agree in $X$. On the other hand, if $Y \notin \mathcal{Y}$, then for every $1 \leq i \leq s$ there exists an attribute $A_{j_{i}} \in Y \backslash Y_{i}$, so whichever element $i \in V D_{A_{0}}$ is put in place of $\perp$ in $t_{0}\left[A_{0}\right]$, we get that $t_{0}[X]=t_{i}[X]$, but $t_{0}[Y] \neq t_{i}[Y]$, hence $T \not \models X \rightarrow_{s p} Y$.

We can characterize the case of fixed right-hand side of an SpFD as well, as it is clear that for a fixed set $Y \subset R$, the collection of attribute sets $\mathcal{X}=\{X: T \models$ $\left.X \rightarrow_{s p} Y\right\}$ forms an up-set, where $T$ is a table over a schema $R$. However, this condition is the only one we have. This is shown in Proposition 8 and Theorem 5 proved in [4]. We repeat them here for the sake of completeness. 
Proposition 8. Let $\left(R, R_{S}\right)$ be a schema, $Y \subset R$ be a fixed set of attributes, furthermore let $\mathcal{X}$ be an upset of subsets of $R \backslash Y$. Then there exists a table $T$ over $\left(R, R_{S}\right)$ such that $T \models X \rightarrow_{s p} Y \Longleftrightarrow X \in \mathcal{X}$.

The proof uses the Armstrong instance construction for strongly possible keys from $[3]$.

Theorem 5. Suppose that $\Sigma=\{s p\langle K\rangle: K \in \mathcal{K}\}$ is a collection of spKey constraints such that if $|K|=1$, then $K \subseteq R_{S}$. Then, there exists an Armstrong table for $\left(R, R_{S}, \Sigma\right)$.

\subsection{Strongly possible functional dependencies axiomatisa- tion}

The first steps towards a possible axiomatisation of spFD's were given in [5]. We studied and analyzed the axioms of weak/strong FD's given by Levene and Loizou [25] and also the axioms of certain FD's given by Köhler and Link [23] in context of spFD's. The investigation showed that some of these axioms are not sound for spFD's. Table 4 shows the axioms that are still sound for spFD's and it also shows several possible weakenings and restrictions that keep soundness for those that are not. All proofs and counterexamples are detailed in $[4,5]$. We repeat them in this paper to be self-contained.

We may conclude from Table 4 that more than one spFD in the premise of a rule usually cause problems in soundness. This is caused by the fact that a single spWorld may not satisfy the different spFD's due to the limitations of visible domains. This problem must be handled for a complete axiomatization. Particularly, the fact that composition rule is not sound in general makes usual proof methods of completeness virtually unusable.

Table 4: spFD Axioms Soundness

\begin{tabular}{|c|c|}
\hline Axiom & spFD Soundness \\
\hline Reflexivity & Sound: If $Y \subseteq X \subseteq R$ then $T \models X \rightarrow_{s p} Y$ \\
\hline Augmentation & Sound: If $T \models X \rightarrow_{s p} Y$ and $W \subseteq R$, then $T \models X W \rightarrow_{s p} Y W$ \\
\hline Union & $\begin{array}{l}\text { Not Sound: If } T \models X \rightarrow_{s p} Y \text { and } T \models X \rightarrow_{s p} Z \text {, } \\
\text { then } T \models X \rightarrow_{s p} Y Z \\
\text { Possible weakenings are: } \\
\text { - Strong FD Mixed-union: } \\
\text { If } T \models \square X \rightarrow Y \text { and } T \models X \rightarrow_{s p} Z \text {, then } T \models X \rightarrow_{s p} Y Z \\
\text { or if } T \models X \rightarrow_{s p} Y \text { and } T \models \square X \rightarrow Z \text {, then } T \models X \rightarrow_{s p} Y Z \\
\text { - Certain FD Mixed-union: } \\
\text { If } T \models X \rightarrow_{s p} Y \text { and } T \models X{ }_{w} \rightarrow Z \text {, then } T \models X \rightarrow_{s p} Y Z \\
\text { - NULL-free union: } \\
\text { If } T \models X \rightarrow_{s p} Y \text { and } T \models X \rightarrow_{s p} Z \text { and } X \subseteq R_{S}, \\
\text { then } T \models X \rightarrow_{s p} Y Z\end{array}$ \\
\hline
\end{tabular}




\begin{tabular}{|c|c|}
\hline Transitivity & $\begin{array}{l}\text { Not Sound: If } T \models X \rightarrow_{s p} Y \text { and } T \models Y \rightarrow_{s p} Z, \\
\text { then } T \models X \rightarrow_{s p} Z \\
\text { Possible weakenings are: } \\
\text { - Strong FD Mixed-transitivity: } \\
\text { If } T \models X \square \rightarrow Y \text { and } T \models Y \rightarrow_{s p} Z \text { or if } T \models X \rightarrow_{s p} Y \text { and } \\
T \models \square Y \rightarrow Z \text {, then } T \models X \rightarrow_{s p} Z \text {. } \\
\text { - Certain FD Mixed-transitivity: } \\
\text { If } T \models X \rightarrow s p Y \text { and } T \models Y w \rightarrow Z \text {, then } T \models X \rightarrow_{s p} Z \\
\text { - Sp-transitivity: } \\
\text { If } T \models X \rightarrow_{s p} Y \text { and } T \models Y \rightarrow_{s p} Z \text {, and } Y \subseteq R_{s}, \\
\text { then } T \models X \rightarrow_{s p} Z\end{array}$ \\
\hline $\begin{array}{l}\text { Pseudo- } \\
\text { transitivity }\end{array}$ & $\begin{array}{l}\text { Not Sound: If } T \models X \rightarrow_{s p} Y \text { and } T \models Y Z \rightarrow_{s p} V \text {, } \\
\text { then } T \models X Z \rightarrow_{s p} V \\
\text { or if } T \models X \rightarrow_{s p} Y Z \text { and } T \models Y \rightarrow_{s p} V \text {, then } T \models X \rightarrow_{s p} Z V \\
\text { Possible weakenings are: } \\
\text { - Strong FD Mixed-pseudo-transitivity: If any of the given } \\
\text { spFD's is strong FD, it is sound. } \\
\text { - Certain FD Mixed-pseudo-transitivity I: } \\
\text { If } T \models X \rightarrow_{s p} Y \text { and } T \models X Y{ }_{w} \rightarrow Z \text {, then } T \models X \rightarrow_{s p} Z \\
\text { - Certain FD Mixed-pseudo-transitivity II: } \\
\text { If } T \models X \rightarrow_{s p} Y \text { and } T \models Y Z{ }_{w} \rightarrow V \text {, then } T \models X Z \rightarrow_{s p} V \\
\text { - NULL-free pseudo-transitivity: If } T \models X \rightarrow_{s p} Y Z \text { and } \\
T \models Y \rightarrow_{s p} V \text { and } Y \subseteq R_{S}, \text { then } T \models X \rightarrow_{s p} Z V \text {. }\end{array}$ \\
\hline Composition & $\begin{array}{l}\text { Not Sound: If } T \models X \rightarrow_{s p} Y \text { and } T \models A \rightarrow_{s p} B \text {, } \\
\text { then } T \models X A \rightarrow_{s p} Y B \\
\text { Possible weakenings are: } \\
\text { - Mixed composition: If } T \models \square X \rightarrow Y \text { and } T \models A \rightarrow_{s p} B \text {, or } \\
\text { if } T \models X \rightarrow_{s p} Y \text { and } T \models \square A \rightarrow B \text {, then } T \models X A \rightarrow_{s p} Y B \text {. } \\
\text { - NULL-free composition: If } T \models X \rightarrow_{s p} Y \text { and } T \models A \rightarrow_{s p} B \\
\text { and } Y A \subseteq R_{S} \text {, then } T \models X A \rightarrow s p Y B \text {. } \\
\text { - Disjoint composition: If } T \models X \rightarrow_{s p} Y \text { and } T \models A \rightarrow_{s p} B \\
\text { and } X \cap A=\emptyset \text {, then } T \models X A \rightarrow_{s p} Y B \text {. }\end{array}$ \\
\hline Decomposition & Sound: If $T \models X \rightarrow_{s p} Y Z$, then $T \models X \rightarrow_{s p} Y$ and $T \models X \rightarrow_{s p} Z$. \\
\hline
\end{tabular}

\subsection{1 sp-keys and sp-FD/c-FDs interaction}

For a relation $R$, the ordinary functional dependency $X \rightarrow R$ implies that $X$ is a key in $R$, because duplicate rows are prohibited in the relational model [9]. On the other hand, for an SQL table $T$, the spFD $X \rightarrow_{s p} T$ does not imply the spKey $s p\langle X\rangle$, because duplicate rows are permitted in the bag semantics of data in SQL tables. 
We study interaction between sp-keys, certain keys, spFD's and c-FDs in the following.

- sp-key/spFD Weakening: $\frac{s p\langle X\rangle}{\left(X \rightarrow_{s p} Y\right)} \forall Y \subseteq R$

Indeed, if there exists an spWorld such that all the tuples are pairwise distinct on $X$, then $X \rightarrow R$ holds in that spWorld.

- certain key/spFD Transitivity: $\frac{\left(X \rightarrow_{s p} Y\right) c\langle X Y\rangle}{s p\langle X\rangle}$

The certain key $c\langle X Y\rangle$ implies that all the tuples are not pairwise weakly similar on $X Y$. Then, for every two tuples, they either have distinct and non-NULL values on $X$ or on $Y$. But we have $X \rightarrow_{s p} Y$, then $\overline{G_{Y}}$ can be list colored by $X$ extensions, and we can extend the extensions of this coloring in any way to $R$. So, by using this coloring, $s p\langle X\rangle$ is satisfied.

However, the sp-key/FD Transitivity true in following form is not sound: $\frac{(X \rightarrow s p Y) s p\langle X Y\rangle}{s p\langle X\rangle}$. The reason is the fact that there will be a possibility of encountering a weak similarity on $X$ and distinctness on $Y$, as illustrated in Table 5 .

Table 5: sp-key/spFD Transitivity dissatisfaction

\begin{tabular}{ccc}
\hline \multicolumn{2}{c}{$\mathbf{X}$} & $\mathbf{Y}$ \\
\hline 1 & 1 & 1 \\
1 & $\perp$ & 1 \\
1 & 2 & 2 \\
\hline
\end{tabular}

\section{3 spFDs for singular attributes}

Substituting a value from the visible domain in place of a $\perp$ produces a duplication in that attribute, since for a singular attribute, the visible domain represents all the possible replacements for any $\perp$ occurrence. For example, in Table 6 , all possible substitutions of the NULL in the second tuple on $X$ are $\{1,2,3\}$, as they form $V D_{A}$. A singular attribute can only be an sp-key if there are no $\perp$ 's in that attribute. However, spFD's are possible between singular attributes with NULL's. Any occurrence of a NULL in the LHS of an spFD for a singular attribute causes a duplication, and this requires a corresponding duplication possibility on the RHS to satisfy the spFD. In the example in Table $6, X \rightarrow_{s p} Y$ holds because, in the first and the third tuples, there is a duplication possibility on $Y$ for the NULL replacement on $X$ in the second tuple. On the other hand, $Y \nrightarrow_{s p} X$ holds, because any replacement for the NULL in the third tuple on $Y$ will not get a corresponding duplication possibility on $X$.

In the present subsection, we study the case of singular attributes as a special case of spFD implication. The following proposition shows a bidirectional property for singular attributes spFD. 
Table 6: spFD for Singular Attributes

\begin{tabular}{cc}
\hline $\mathbf{X}$ & $\mathbf{Y}$ \\
\hline 1 & 1 \\
$\perp$ & 1 \\
2 & $\perp$ \\
3 & 2 \\
\hline
\end{tabular}

Proposition 9. For singular attributes $X$ and $Y$, if $T \models X \rightarrow_{s p} Y$ and $\left|V D_{X}\right|=$ $\left|V D_{Y}\right|$, then $T \mid=Y \rightarrow_{s p} X$

Proof. We may assume without loss of generality that $V D_{X}=\{1,2, \ldots \ell\}$. Since $T \models X \rightarrow_{s p} Y$, for every $i \in\{1,2, \ldots \ell\}$ if $t \in T$ is a tuple, then $t[X]=i$ implies that $t[Y]=a_{i}$ or $t[Y]=\perp$. Also, let $\left\{b_{1}, b_{2}, \ldots, b_{r}\right\} \subset V D_{Y}$ be those pairwise distinct visible domain values that satisfy $t[Y]=b_{j} \Rightarrow t[X]=\perp$. Assume that for $1 \leq i \leq k$ we have non-NULL $a_{i}$ such that there exists a tuple $t$ with $t[X]=i$ and $t[Y]=a_{i}$, that is, for $k+1 \leq j \leq \ell$ we have $t[X]=j \Rightarrow t[Y]=\perp$. Table 7 shows the possible types of tuples on $\{X, Y\}$ If $a_{i} \neq a_{j}$ for $i \neq j$, then by $\left|V D_{X}\right|=\left|V D_{Y}\right|$ we have that $r=\ell-k$ and the NULL's can easily be substituted in these two columns so that $1 \leq i \leq k$ is matched with $a_{i}$ and $k+1 \leq j \leq \ell$ is matched with $b_{j-k}$ so the two columns are basically identical, that is $Y \rightarrow_{s p} X$ holds. On the other hand, if $a_{i}=a_{j}$ for some $i \neq j$, then we have that $r>\ell-k$ since the sizes of divisible domains of $X$ and $Y$ are the same. Consider a spWorld $T^{\prime}$ of $T$ that satisfies $T^{\prime} \models X \rightarrow Y$ functional dependency. Such a $T^{\prime}$ exists, since $T \models X \rightarrow_{s p} Y$. Now, the NULL's in the tuples that have value $b_{j}$ in $Y$ can only be substituted by values from $\{k+1, \ldots \ell\}$, because for $1 \leq i \leq k$ there are tuples with non-NULL values $t[X]=i$ and $t[Y]=a_{i}$. However, as $r>\ell-k$, we must have $1 \leq u<v \leq r$ and $k+1 \leq j \leq \ell$ so that we have two tuples $t, t^{\prime}$ with $t[X]=t^{\prime}[X]=j$ and $t[Y]=b_{u}$ and $t^{\prime}[Y]=b_{v}$ contradicting to $T^{\prime} \models X \rightarrow Y$.

Example 2. The table below shows an instance $T$ with $T \models X \rightarrow_{s p} Y$ and $\left|V D_{X}\right|=\left|V D_{Y}\right|=2$. Value 2 in attribute $X$ is shown only in $t_{4}$ where $t_{4}[Y]=\perp$. Then, to have $\left|V D_{X}\right|=\left|V D_{Y}\right|$, we have value $a_{2}$ in attribute $Y$ is shown only in tuples having $\perp$ on $X$. Then, $X$ and $Y$ are two singular attributes satisfy a bidirectional implication property.

Proposition 10. For singular $X$ and $Y$, if $T \models X \rightarrow_{s p} Y$, then $\left|V D_{X}\right| \geq\left|V D_{Y}\right|$.

Proof. Let $T^{\prime}$ be a spWorld of $T$ that satisfies functional dependency $X \rightarrow Y$ that exists by $T \models X \rightarrow_{s p} Y$. Observe that the set of values appearing in column $X$ of $T^{\prime}$ is $V D_{X}$, while that of values appearing in column $Y$ is $V D_{Y}$. Since if $t_{1}^{\prime}[X]=t_{2}^{\prime}[X]$ implies $t_{1}^{\prime}[Y]=t_{2}^{\prime}[Y]$ for any $t_{1}^{\prime}, t_{2}^{\prime} \in T^{\prime}$, the mapping $f: V D_{Y} \rightarrow V D_{X}$ given by $f\left(v^{\prime}\right)=v$ if there is a tuple $t^{\prime} \in T^{\prime}$ such that $t^{\prime}[X]=v, t^{\prime}[Y]=v^{\prime}$ is well defined and is an injection. 
In the next proposition, we show the bidirectional implication of the spFD in the singular attributes directed graph of spFD's.

Proposition 11. Let $T$ be an instance over the relation $R$. If the spFD's between singular attributes $X_{i} \in R$, for $i=1,2 \ldots, w$, form a directed circle in the spFD graph, that is $T \models X_{i} \rightarrow_{s p} X_{i+1}$ and $T \models X_{w} \rightarrow_{s p} X_{1}$. Then the reverse direction of the spFD circle also holds in $T$, i.e. $T \models X_{i+1} \rightarrow_{s p} X_{i}$ and $T \models X_{1} \rightarrow_{s p} X_{w}$.

Proof. As the spFD's form a circle in the spFD graph, then all the attributes have the same number of values in their visible domains. Indeed, by $T \models X_{i} \rightarrow_{s p}$ $X_{i+1}$, we have $\left|V D_{X_{i}}\right| \geq\left|V D_{X_{i+1}}\right|$ for any $i$, and by $T \models X_{w} \rightarrow_{s p} X_{1}$, we have $\left|V D_{X_{w}}\right| \geq\left|V D_{X_{1}}\right|$. So, $\left|V D_{X_{i}}\right|=\left|V D_{X_{i+1}}\right|$ for any $i$. Hence, by Proposition 9 , the other direction for each spFD is also satisfied by $T$.

The bidirectional implication of the spFD between the singular attributes does not show that the complements of their weak similarity graphs are the same, For example, $Y \rightarrow_{s p} Z$ and $Z \rightarrow_{s p} Y$ hold in the table below, but $\overline{G_{Y}} \neq \overline{G_{Z}}$. On the

Table 7: Possible types of tuples

\begin{tabular}{c|c}
$X$ & $Y$ \\
\hline 1 & $a_{1}$ \\
1 & $\perp$ \\
\hline 2 & $a_{2}$ \\
2 & $\perp$ \\
\hline$\vdots$ & $\vdots$ \\
\hline$k$ & $a_{k}$ \\
$k$ & $\perp$ \\
\hline$k+1$ & $\perp$ \\
$\vdots$ & $\vdots$ \\
$\ell$ & $\perp$ \\
\hline$\perp$ & $a_{1}$ \\
$\vdots$ & $\vdots$ \\
$\perp$ & $a_{k}$ \\
$\perp$ & $b_{1}$ \\
$\vdots$ & $\vdots$ \\
$\perp$ & $b_{r}$ \\
\hline &
\end{tabular}




\begin{tabular}{lll}
\cline { 2 - 3 }$t_{1}$ & $\mathbf{X}$ & $\mathbf{Y}$ \\
\cline { 2 - 3 }$t_{2}$ & $\perp$ & $a_{2}$ \\
$t_{3}$ & 1 & $a_{1}$ \\
$t_{4}$ & 2 & $\perp$ \\
$t_{5}$ & $\perp$ & $a_{2}$ \\
$t_{6}$ & $\perp$ & $\perp$ \\
\cline { 2 - 3 }
\end{tabular}

other hand, $X \rightarrow_{s p} Y$ and $X \rightarrow_{s p} Z$ hold, but $X \nrightarrow_{s p} Y Z$.

\begin{tabular}{ccc}
\hline $\mathrm{X}$ & $\mathrm{Y}$ & $\mathrm{Z}$ \\
\hline 1 & 1 & $\perp$ \\
$\perp$ & 2 & 1 \\
2 & $\perp$ & 2 \\
\hline
\end{tabular}

Propositions 9 and 11 allow us to introduce the following rule.

Digraph rule Let $G=(R, E)$ be a directed graph If $\left(A_{i}, A_{j}\right) \in E$ and $A_{i}$ and $A_{j}$ are in the same strongly connected component of $G$, then $\left(A_{j}, A_{i}\right) \in E$ holds, as well.

Theorem 6. Let $T$ be an $S Q L$ table over scheme $R=\left\{A_{1}, A_{2}, \ldots, A_{n}\right\}$. If $G=$ $(R, E)$ is a directed graph defined by $\left(A_{i}, A_{j}\right) \in E \Longleftrightarrow T \models\left\{A_{i}\right\} \rightarrow_{s p}\left\{A_{j}\right\}$ for attributes $A_{i}, A_{j} \in R$, then $G=(R, E)$ satisfies the Digraph rule. Furthermore, for every directed graph $G=(R, E)$ that satisfies the Digraph rule there exists an $S Q L$ table $T$ such that $\left(A_{i}, A_{j}\right) \in E \Longleftrightarrow T \models\left\{A_{i}\right\} \rightarrow_{s p}\left\{A_{j}\right\}$.

The proof of Theorem 6 is based on a series of propositions.

Proposition 12. Let $T$ be an $S Q L$ table over scheme $R=\left\{A_{1}, A_{2}, \ldots, A_{n}\right\}$ and let $G=(R, E)$ be the directed graph defined by $\left(A_{i}, A_{j}\right) \in E \Longleftrightarrow T \models\left\{A_{i}\right\} \rightarrow_{s p}\left\{A_{j}\right\}$ for attributes $A_{i}, A_{j} \in R$. Then $G=(R, E)$ satisfies the Digraph rule.

Proof. Let $\left(A_{i}, A_{j}\right) \in E$ be an edge of the directed graph defined by spFD's between singular attributes so that $A_{i}$ and $A_{j}$ are in the same strongly connected component of $G$. This means that there exists a directed path $A_{j}=X_{1}, X_{2}, \ldots X_{w}=A_{i}$ in $G$, thus together with edge $\left(A_{i}, A_{j}\right)$ a directed cycle is obtained. So by Proposition 11 , the other direction of the spFD's hold, i.e $T \models A_{j} \rightarrow_{s p} A_{i}$. 
Proposition 13. Let $G=(R, E)$ be a strongly connected graph that satisfies the Digraph rule. Then there exists an $S Q L$ table $T$ over schema $R=\left\{A_{1}, A_{2}, \ldots, A_{n}\right\}$ such that $\left(A_{i}, A_{j}\right) \in E \Longleftrightarrow T \models\left\{A_{i}\right\} \rightarrow_{s p}\left\{A_{j}\right\}$. Furthermore, we may assume that $V D\left(A_{i}\right)=\{1,2\}$ for all $i=1,2, \ldots, n$ in $T$ and that if $\left(A_{i}, A_{j}\right) \notin E$, then there exists two rows of $T$ that agree and non-null in $A_{i}$ and differ and non-null in $A_{j}$

Proof. Note that a strongly connected graph $G=(R, E)$ satisfies the Digraph rule iff $\left(A_{i}, A_{j}\right) \in E \Longleftrightarrow\left(A_{j}, A_{i}\right) \in E$. In particular, for any induced subgraph $G^{\prime}=$ $\left(R^{\prime}, E^{\prime}\right)$ of $G=(R, E)$ also satisfies the Digraph rule. We may assume without loss of generality that $\left(A_{1}, A_{2}\right) \in E$. Let $G_{k}=\left(R_{k}, E_{k}\right)$ be the subgraph of $G=(R, E)$ induced by $R_{k}=\left\{A_{1}, A_{2}, \ldots, A_{k}\right\}$. We use induction on $k$ to prove that there exists an SQL table $T_{k}$ over $R_{k}$ such that $\left(A_{i}, A_{j}\right) \in E_{k} \Longleftrightarrow T_{k}=\left\{A_{i}\right\} \rightarrow{ }_{s p}\left\{A_{j}\right\}$ and if $\left(A_{i}, A_{j}\right) \notin E$, then there exists two rows of $T_{k}$ that agree and non-null in $A_{i}$ and differ and non-null in $A_{j}$. The base case $k=2$ is trivial

$$
T_{2}=\begin{array}{|c|c|}
\hline A_{1} & A_{2} \\
\hline 1 & 1 \\
2 & \perp \\
\perp & 2 \\
\hline
\end{array}
$$

so $T_{2}=\left\{t_{0}, t_{1}, t_{2}\right\}$ with $t_{0}\left[A_{j}\right]=1$ for $j \in\{1,2\}$ and

$$
t_{i}\left[A_{j}\right]=\left\{\begin{array}{cc}
2 & \text { if } i=j \\
\perp & \text { if } i \neq j
\end{array} .\right.
$$

Note that $T_{2}$ has one entry 2 in each column, and these entries are in different rows, in column $A_{i}$ the 2 is in row $t_{i}$. Now assume that $T_{k}=\left\{t_{0}, t_{1}, t_{2}, \ldots, t_{k}\right\}$ exists for $G_{k}=\left(R_{k}, E_{k}\right)$ such that column $A_{i}$ has its only entry 2 in row $t_{i}$ and consider $G_{k+1}$. We add new column $A_{k+1}$ and a new row (tuple) $t_{k+1}$ to $T_{k}$ as follows.

$$
t_{i}\left[A_{k+1}\right]=\left\{\begin{array}{ll}
1 & \text { if }\left(A_{k+1}, A_{i}\right) \notin E_{k+1} \\
\perp & \text { if }\left(A_{k+1}, A_{i}\right) \in E_{k+1}
\end{array} \text { for } i=1,2, \ldots, k \text { and } t_{k+1}\left[A_{k+1}\right]=2\right.
$$

Furthermore

$$
t_{k+1}\left[A_{i}\right]=\left\{\begin{array}{ll}
1 & \text { if }\left(A_{i}, A_{k+1}\right) \notin E_{k+1} \\
\perp & \text { if }\left(A_{i}, A_{k+1}\right) \in E_{k+1}
\end{array} \text { for } i=1,2, \ldots, k\right.
$$




$T_{k+1}=$\begin{tabular}{|c|c|c|c|c|}
\hline$A_{1}$ & $A_{2}$ & $\ldots$ & $A_{k}$ & $A_{k+1}$ \\
\hline 1 & 1 & $1 \ldots 1$ & 1 & 1 \\
\hline 2 & $\perp$ & $1 \ldots 1$ & 1 & 1 \\
\hline$\perp$ & 2 & $1 \ldots \perp$ & 1 & $\perp$ \\
\hline 1 & $\perp$ & $2 \ldots 1$ & $\perp$ & $\perp$ \\
\hline$\vdots$ & $\vdots$ & $\ddots$ & $\vdots$ & $\vdots$ \\
\hline$\perp$ & 1 & $1 \ldots \perp$ & 2 & 1 \\
\hline 1 & $\perp$ & $1 \ldots \perp$ & 1 & 2 \\
\hline
\end{tabular}

This table satisfies the requirements, since no $A_{i} \rightarrow_{s p} A_{j}$ for $1 \leq i, j \leq k$ is destroyed by row $t_{k+1}$ by imputing 1's in place of $\perp$ 's in the last row. Similarly, if we impute a 2 in place of $\perp$ in the last column in row $t_{i}$ and everywhere else 1 's in that column, then we get a strongly possible world showing $A_{k+1} \rightarrow_{s p} A_{i}$. Furthermore imputing 2 in place of $\perp$ 's in $t_{k+1}$ we get $A_{i} \rightarrow_{s p} A_{k+1}$ exactly if $\left(A_{i}, A_{k+1}\right) \in E_{k+1}$.

Proof. (of Theorem 6). We use induction on the number $t$ of strongly connected components of $G=(R, E)$. The base case $t=1$ is the statement of Proposition 13 . Let the strongly connected components of $G=(R, E)$ be $C_{1}, C_{2}, \ldots, C_{t}$, we may assume without loss of generality, that they are in a topological sort order, that is if $\left(A_{i}, A_{j}\right) \in E$ and $A_{i} \in C_{i}$ and $A_{j} \in C_{j}$, then $i \leq j$. There exists an SQL table $T_{i}$ for each $C_{i}$ such that $\{A, B\} \in E\left(C_{i}\right) \Longleftrightarrow T_{i} \models A \rightarrow_{s p} B$. Furthermore, by Proposition 13 we have that entries in $T_{i}$ are $1,2, \perp$, if $A \rightarrow_{s p} B$, 1 's in column $A$ match 1's or $\perp$ in $B$, and 2's are not in the same row with a 1 in these 2 columns. If $A t_{s p} B$, then there exist two rows as following:

\begin{tabular}{cc}
$A$ & $B$ \\
\hline 1 & 1 \\
1 & 2
\end{tabular}

Assume by induction, we have a table $T^{t-1}$ for the subgraph induced by $C_{1} \cup$ $C_{2} \cup \ldots \cup C_{t-1}$. Construct $T^{t}$ as follows. First position $T^{t-1}$ and $T_{t}$ on disjoint row and column sets so that $T^{t-1}$ is extended by 1's in the columns of $C_{t}$. In the rows of $T_{t}$ put matrix $M\left(r_{1}, r_{2}, \ldots, r_{k}\right)$, which has entry $r_{i}$ in its $i^{\text {th }}$ row for each column of $C_{1} \cup C_{2} \cup \ldots \cup C_{t-1}$, where $r_{1}, r_{2}, \ldots r_{k}$ are pairwise distinct numbers, different from anything appearing in $T^{t-1}$ : 


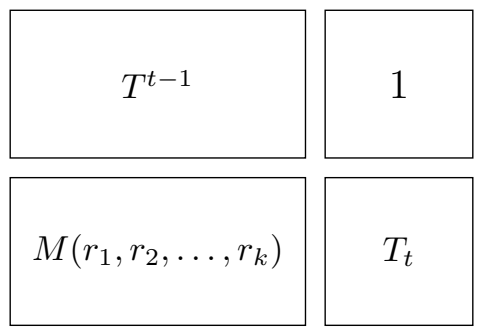

Then we add two rows, $\mathbf{t}_{\mathbf{1}}^{\mathbf{A}}$ and $\mathbf{t}_{\mathbf{2}}^{\mathbf{A}}$ for each $A \in C_{1} \cup C_{2} \cup \ldots \cup C_{t-1}$ as follows, $\forall B \in C_{1} \cup C_{2} \cup \ldots \cup C_{t-1}$ and for all $X, Y \in C_{t}$ if $A \rightarrow_{s p} X$ and $A \rightarrow_{s p} Y$.

\begin{tabular}{c|cccc} 
& $A$ & $B$ & $X$ & $Y$ \\
\hline $\mathbf{t}_{\mathbf{1}}^{\mathbf{A}}$ & $r_{A}$ & $\perp$ & $\perp$ & 1 \\
$\mathbf{t}_{\mathbf{2}}^{\mathbf{A}}$ & $r_{A}$ & $\perp$ & $\perp$ & 2
\end{tabular}

where $r_{A}$ is a new value not appearing in $T^{t-1}$, also $r_{A} \neq r_{i} \forall i=1,2, \ldots, k$ and if $A \neq B$ then $r_{A} \neq r_{B}$.

Let $T^{t}$ be the table obtained. We check pairs of attributes $A, X$ that $T^{t} \models$ $A \rightarrow s p X \Longleftrightarrow(A, X) \in E$.

Case 1: Both $A, X \in C_{1} \cup C_{2} \cup \ldots \cup C_{t-1}$. If $(A, X) \notin E$, then by the induction hypothesis there exist two rows of $T^{t-1}$ that are both not null in both $A$ and $X$ and agree in $A$ and differ in $X$, so $T^{t} \not \models A \rightarrow_{s p} X$. On the other hand, if $(A, X) \in E$, then the induction hypothesis provides an spWorld $T^{*}$ of $T^{t-1}$ that $T^{*} \models A \rightarrow X$. Columns $A$ and $X$ can be extended identically below $T^{t-1}$ so that values on those rows in $A$ and $X$ differ from values in $T^{*}$, thus imputing any values in place of nulls in the other columns we get an spWord $T^{\prime}$ of $T^{t}$ such that $T^{\prime} \models A \rightarrow X$.

Case 2: Both $A, X \in C_{t}$. If $(A, X) \notin E$, then by Proposition 13 there are two rows of $T_{t}$ that they are both 1 in $A$ and take different values (1 and 2) in $X$, so $T^{t} \not \models A \rightarrow_{s p} X$. On the other hand, if $(A, X) \in E$, then there is an spWord of $T_{t}$ that has columns $A$ and $X$ identical. The rows above $T_{t}$ are also identical in $A$ and $X$ and these two columns can be extended identically below $T_{t}$, as well so that 1 's in $A$ match 1's in $X$ and the same holds for entries 2. Then other null values of $T^{t}$ can be arbitrarily substituted from visible domains of the given attributes and an spWord is obtained where $A \rightarrow X$ holds, that is $T^{t} \models A \rightarrow_{s p} X$.

Case 3: $A \in C_{1} \cup C_{2} \cup \ldots \cup C_{t-1}$ and $X \in C_{t}$. In this case $(X, A) \notin E$ holds, and there are two rows in $T^{t-1}$ that have different not null values in $A$, but these two rows take value 1 in $X$, so $T^{t} \forall X \rightarrow_{s p} A$. If If $(A, X) \notin E$, then rows $\mathbf{t}_{\mathbf{1}}^{\mathbf{A}}$ and $\mathbf{t}_{\mathbf{2}}^{\mathbf{A}}$ have identical values in $A$ but different values in $X$, so $T^{t} \not \models A \rightarrow_{s p} X$. On the other hand, if $(A, X) \in E$, then the nulls of $X$ in rows $\mathbf{t}_{\mathbf{1}}^{\mathbf{A}}$ and $\mathbf{t}_{\mathbf{2}}^{\mathbf{A}}$ can be substituted by 1 's, similarly for any rows $\mathbf{t}_{\mathbf{1}}^{\mathbf{B}}$ and $\mathbf{t}_{\mathbf{2}}^{\mathbf{B}}$ that contain nulls in $X$. If for some $B \in C_{1} \cup C_{2} \cup \ldots \cup C_{t-1}$ the corresponding rows $\mathbf{t}_{1}^{\mathbf{B}}$ and $\mathbf{t}_{\mathbf{2}}^{\mathbf{B}}$ contain 1 and 2 in column $X$ (i.e. $(B, X) \notin E)$, then in $\mathbf{t}_{\mathbf{1}}^{\mathbf{B}}$ we substitute 1 in column $A$, 
and in $\mathbf{t}_{\mathbf{2}}^{\mathbf{B}}$ substitute $r_{i}$ in place of the null in column $A$, where $r_{i}$ is the value in $M\left(r_{1}, r_{2}, \ldots, r_{k}\right)$ that stands in the unique row of $T_{t}$ which has a 2 in column $X$. The rest of the nulls of $T^{t}$ can be imputed arbitrarily from the appropriate visible domains, the spWord of $T_{t}$ obtained satisfies functional dependency $A \rightarrow X$.

\section{Conclusions}

Entering incomplete tuples are allowed into many recent systems' databases. Several research work studied the keys and functional dependencies constraints over incomplete data, such as p/c-keys [22], spKeys [3], c-FDs [23], p-FDs[26], and s/wFDs [25].

This paper continued the research work started in [3] of strongly possible worlds of SQL tables with NULL's. We introduced a polynomial-time algorithm that checks whether a given set $K$ of attributes is a spKey or not. We also proved that it is NPcomplete to do the same for an arbitrary system of $\Sigma=\left\{s p\left\langle K_{i}\right\rangle: i=1,2, \ldots n\right\}$ of spKey constraints. On the other hand, we showed that $\Sigma$ can be verified in polynomial time if the sets $K_{i}$ are pairwise disjoint. Further, we studied strongly possible functional dependencies that were introduced in [4] earlier, as a functional dependency constraint in an SQL table containing NULL's, using the visible domain concept. A graph-theoretical characterization using list coloring is given that can be employed to check when a given spFD holds. This characterization allowed us to prove that verifying whether a single spFD $X \rightarrow_{s p} Y$ holds is NP-complete. This is in sharp contrast with spKey problem, where a single key can be checked in polynomial time.

In another paper [5] spFD properties and axioms analogous to those of weak and strong FD's given by Levene et.al. are introduced and suitable weakenings were given for those axioms that are non sound for spFD's. Here we summarize those in a table for completeness and extend the investigation on sp-Keys and sp/c-FDs together to obtain weakening and transitivity interaction rules.

Our study shows that the spFD's between singular attributes have special properties, because the visible domain represents all the possible extensions for any NULL occurrence. There is a natural correspondence between directed graphs and single attribute spFD's, a characterization of those directed graphs that may occur in this context was given.

The properties of spFD's listed form a step toward a possible axiomatization of spFD's. The main challenge of applying the visible domain is that different spFD's in the premises of rules may hold in different and incompatible strongly possible worlds. More investigation is needed to find how to incorporate this into the axiom system. A first step would be to resolve the following open problem.

Question 1. Let us assume that $R_{S}=\emptyset$. Do Reflexivity, Augmentation, Decomposition, Disjoint composition, and Digraph rule form a complete system of inference rules for strongly possible functional dependencies in this case?

A further simplification that could be attacked using Theorem 3 is when we 
assume that there exist no spFD's between singleton attributes. Our experience shows that in that case spFD's are mostly independent of each other, so the first four rules of the previous question could be a complete set for inferences. Another future research direction is defining the closures concerning spFD's. Where the usual definition of $X^{+}=\left\{A: T \models X \rightarrow_{s p} A\right\}$ may result in $T \not \models X \rightarrow_{s p} X^{+}$for spFD's, as the union rule is not sound.

Finally, for database tables, lossless decomposition is an important application of FD's to eliminate redundancy and the possibilities of inconsistent updates. Köhler and Link[23] and Wei and Link [28] show how to use c-FD's or embedded FD's for that. Our future work will include a similar investigation for spFD's.

\section{References}

[1] Achs, Ágnes and Kiss, Attila. Fuzzy extension of datalog. Acta Cybernetica, 12(2):153-166, 1995. URL: https://cyber.bibl.u-szeged.hu/index.php/ actcybern/article/view/3454.

[2] Acuna, Edgar and Rodriguez, Caroline. The treatment of missing values and its effect on classifier accuracy. In Classification, clustering, and data mining applications, pages 639-647. Springer, 2004. DOI: 10.1007/ 978-3-642-17103-1_60.

[3] Alattar, Munqath and Sali, Attila. Keys in relational databases with nulls and bounded domains. In European Conference on Advances in Databases and Information Systems, pages 33-50. Springer, 2019. DOI: 10.1007/ 978-3-030-28730-6_3.

[4] Alattar, Munqath and Sali, Attila. Functional dependencies in incomplete databases with limited domains. In International Symposium on Foundations of Information and Knowledge Systems, pages 1-21. Springer, 2020. DOI: 10.1007/978-3-030-39951-1_1.

[5] Alattar, Munqath and Sali, Attila. Towards an axiomatization of strongly possiblefunctional dependencies. The Vietnam Journal of Computer Science, 8(1):133-151, 2021. DOI: 10.1142/S2196888821500056.

[6] Atzeni, Paolo and Morfuni, Nicola M. Functional dependencies and constraints on null values in database relations. Information and Control, 70(1):1-31, 1986. DOI: $10.1016 / \mathrm{S} 0019-9958(86) 80022-5$.

[7] Chang, Gang and Ge, Tongmin. Comparison of missing data imputation methods for traffic flow. In Proceedings 2011 International Conference on Transportation, Mechanical, and Electrical Engineering (TMEE), pages 639-642. IEEE, 2011. DOI: 10.1109/TMEE.2011.6199284.

[8] Cheng, Ching-Hsue, Wei, Liang-Ying, and Lin, Tzu-Cheng. Improving relational database quality based on adaptive learning method for estimating 
null value. In Second International Conference on Innovative Computing, Informatio and Control (ICICIC 2007), pages 81-81. IEEE, 2007. DOI: 10.1109/ICICIC. 2007.350 .

[9] Codd, Edgar F. The relational model for database management: version 2. Addison-Wesley Longman Publishing Co., Inc., 1990.

[10] Date, CJ. Not is not "not"! (notes on three-valued logic and related matters), chapter 8. In Relational Database Writings. Addison-Wesley, Reading, MA, 1989.

[11] Dhevi, AT Sree. Imputing missing values using inverse distance weighted interpolation for time series data. In 2014 Sixth International Conference on Advanced Computing (ICoAC), pages 255-259. IEEE, 2014. DOI: 10.1109/ ICoAC. 2014.7229721.

[12] Farhangfar, Alireza, Kurgan, Lukasz, and Dy, Jennifer. Impact of imputation of missing values on classification error for discrete data. Pattern Recognition, 41(12):3692-3705, 2008. DOI: 10.1016/j ·patcog.2008.05.019.

[13] Farhangfar, Alireza, Kurgan, Lukasz A, and Pedrycz, Witold. Experimental analysis of methods for imputation of missing values in databases. In Intelligent Computing: Theory and Applications II, volume 5421, pages 172-182. International Society for Optics and Photonics, 2004. DOI: 10.1117/12.542509.

[14] Farhangfar, Alireza, Kurgan, Lukasz A, and Pedrycz, Witold. A novel framework for imputation of missing values in databases. IEEE Transactions on Systems, Man, and Cybernetics-Part A: Systems and Humans, 37(5):692-709, 2007. DOI: 10.1109/TSMCA. 2007.902631.

[15] Grahne, Gösta. Dependency satisfaction in databases with incomplete information. In Proceedings of the 10th International Conference on Very Large Data Bases, pages 37-45, 1984.

[16] Grzymala-Busse, Jerzy W. and Hu, Ming. A comparison of several approaches to missing attribute values in data mining. In International Conference on Rough Sets and Current Trends in Computing, pages 378-385. Springer, 2000.

[17] Hartmann, Sven and Link, Sebastian. The implication problem of data dependencies over SQL table definitions: Axiomatic, algorithmic and logical characterizations. ACM Trans. Database Syst., 37(2), 2012. DOI: $10.1145 / 2188349.2188355$.

[18] Imielinski, Tomasz. Incomplete information in logical databases. IEEE Data Engineering Bulletin, 12(2):29-40, 1989.

[19] Imielinski, Tomasz and Lipski, Witold. Incomplete information in relational databases. Journal of the ACM, 31(4):761-791, 1984. DOI: 10.1145/1634. 1886. 
[20] Jansen, Klaus and Scheffler, Petra. Generalized coloring for tree-like graphs. Discrete Applied Mathematics, 75(2):135-155, 1997. DOI: 10.1016/ S0166-218X (96)00085-6.

[21] Kiss, A. and Márkus, T. Functional and inclusion dependencies and their implication problems. In Proceedings of the 10th International Seminar on Database Management Systems, pages 31-38, 1987.

[22] Köhler, Henning, Leck, Uwe, Link, Sebastian, and Zhou, Xiaofang. Possible and certain keys for SQL. The VLDB Journal, 25(4):571-596, 2016. DOI: 10.1007/s00778-016-0430-9.

[23] Köhler, Henning and Link, Sebastian. SQL schema design: Foundations, normal forms, and normalization. Information Systems, 76:88-113, 2018. DOI: $10.1016 / j$.is. 2018.04 .001 .

[24] Kratochvil, Jan and Tuza, Zsolt. Algorithmic complexity of list colorings. Discrete Applied Mathematics, 50(3):297-302, 1994. DOI: 10.1016/ 0166-218X (94) 90150-3.

[25] Levene, Mark and Loizou, George. Axiomatisation of functional dependencies in incomplete relations. Theoretical Computer Science, 206(1):283-300, 1998. DOI: $10.1016 /$ S0304-3975(98)80029-7.

[26] Lien, Y Edmund. On the equivalence of database models. Journal of the ACM, 29(2):333-362, 1982. DOI: 10.1145/322307.322311.

[27] Vassiliou, Yannis. Functional dependencies and incomplete information. In Proceedings of the 6th VLDB Conference. Morgan Kaufmann Publishers, 1980.

[28] Wei, Ziheng and Link, Sebastian. Embedded functional dependencies and datacompleteness tailored database design. Proceedings of the VLDB Endowment, 12(11):1458-1470, 2019. DOI: 10.14778/3342263.3342626.

[29] Zhang, Shichao, Qin, Zhenxing, Ling, Charles X, and Sheng, Shengli. "Missing is useful": Missing values in cost-sensitive decision trees. IEEE Transactions on Knowledge and Data Engineering, 17(12):1689-1693, 2005. DOI: 10.1109/ TKDE. 2005.188. 